\title{
Birth Preparedness, Complication Readiness, and Determinants Among Women Attending Antenatal Care from Ethiopia: A Cross-sectional Facility-Based Survey
}

\author{
Alemayehu Dereje Jaleta ${ }^{1}$, Legesse Tadesse ${ }^{2}$, Hiwot Zelalem ${ }^{2}$, Dassalegn Daraje Jalata ${ }^{3}$, \\ ${ }^{1}$ Department of Nursing, Sibu Sire Health Center, Sire, Ethiopia \\ ${ }^{2}$ Department of Public Health, Arsi University, Assela, Ethiopia \\ ${ }^{3}$ Department of Food Science and Nutrition Research, Ethiopian Institute of Agricultural Research, Addis Ababa, Ethiopia \\ Email address: \\ alemadereje2020@gmail.com (A. D. Jaleta), legesset2008@gmail.com (L. Tadesse), Hiwet58@yahoo.com (H. Zelalem), \\ dessdrj@gmail.com (D. D. Jalata) \\ ${ }^{*}$ Corresponding author
}

To cite this article:

Alemayehu Dereje Jaleta, Legesse Tadesse, Hiwot Zelalem, Dassalegn Daraje Jalata. Birth Preparedness, Complication Readiness, and Determinants Among Women Attending Antenatal Care from Ethiopia: A Cross-sectional Facility-Based Survey. European Journal of Clinical and Biomedical Sciences. Vol. 7, No. 3, 2021, pp. 37-43. doi: 10.11648/j.ejcbs.20210703.11

Received: April 19, 2021; Accepted: May 27, 2021; Published: June 4, 2021

\begin{abstract}
A large number of mothers die from pregnancy complications and childbirth worldwide and Sub-Saharan Africa including Ethiopia accounts for the third. Although several studies were done on birth preparedness and complication readiness in different parts, there is limited information in the current study setting. Hence, the aim of the current study was to assess birth preparedness and complication readiness practices and determinants among pregnant women attending antenatal care in Sibu Sire District, East Wollega Zone, Ethiopia. Accordingly, a facility-based cross-sectional study was conducted on 398 women attending ANC. Data were collected through an interviewer applied structured questionnaire. Data were entered into EPI-INFO version 7 and exported to SPSS version 21 for analysis. After the descriptive statistics, bivariate and multiple variable regression were carried out using the odds ratio and its $95 \%$ confidence interval at $\mathrm{p}<0.05$. The response rate was $100 \%$ and the magnitude of BPCR among pregnant women in the study setting was $30.2 \%$. Educated elementary, achieved secondary and above, history of live-birth, history of stillbirth, ANC four \& above visits, knowledge of danger signs of pregnancy, awareness of BPCR, and knowledge of birth preparedness were significantly associated with BPCR with $(\mathrm{AOR}=2.59 ; 95 \% \mathrm{CI}: 1.30-5.18),(\mathrm{AOR}=5.88 ; 95 \% \mathrm{C} / \mathrm{I}: 2.57-13.46),(\mathrm{AOR}=4.31 ; 95 \% \mathrm{C} / \mathrm{I}: 2.45-7.60)$, $(\mathrm{AOR}=2.00 ; 95 \% \mathrm{C} / \mathrm{I}: 1.01-3.86),(\mathrm{AOR}=2.11 ; 95 \% \mathrm{C} / \mathrm{I}: 1.25-3.55),(\mathrm{AOR}=2.21 ; 95 \% \mathrm{C} / \mathrm{I}: 1.17-4.18),(\mathrm{AOR}=2.55 ; 95 \%$ CI: 1.48-4.40), (AOR=2.1; 95\% C/I: 2.1 (1.24-3.53)) respectively. In conclusion, the magnitude of BPCR practice was low in the area. The key determinants in the study setting were educational status, history of live-birth, history of stillbirth, having ANC $\geq 4$ visits, knowledge of danger signs of pregnancy, awareness of BPCR, and knowledge of BPCR. Therefore, improving the means of creating awareness, ANC attendance, and education coverage are recommended based on the finding. Furthermore, to explore the underlined reason a wide scope follow-up study and mixed methods studies is recommended.
\end{abstract}

Keywords: ANC, Birth Preparedness, Complication Readiness, Knowledge, Sire

\section{Introduction}

Birth Preparedness and Complication Readiness (BPCR) is a strategy to promote the timely use of skilled maternal health care, especially starting from pregnancy, labor, delivery, and immediate postpartum period identified as comprehensive strategies to address high maternal morbidity and mortality [1]. Birth preparedness and complication readiness counseling are expected to provide during antenatal care visits [2].

Globally, estimates of 303,000 women die each year during and following pregnancy and childbirth. About $99 \%$ 
$(302,000)$ of maternal deaths account for developing regions, with Sub-Saharan alone accounting for roughly a third followed by South Asia [3]. In Ethiopia, studies indicated maternal mortality remained stagnant. Ethiopia Demographic and health surveys (EDHS) of the last two decades show the maternal mortally ratio per 100,000 live births in 2000, 2005, 2011, and 2016 were 871, 673, 676, and 412 respectively [4].

According to WHO (2016), an estimate of $78 \%$ of pregnant women was attended by a skilled birth attendant during childbirth, $58 \%$ of them had four or more antenatal care visits, and only $52 \%$ received postpartum care [3]. In Ethiopia, a key factor contributing to high maternal mortality is the low rate of skilled care during pregnancy and delivery. The Ethiopian Demographic and Health Survey of 2016 showed that only $62 \%$ of pregnant women received ANC at least once and approximately $28 \%$ of births were assisted by skilled birth attendants. About half $(50.7 \%)$ of pregnant women received ANC at least once and $22 \%$ had received four or more ANC in Oromia regional state [4].

The most causes of maternal deaths are postpartum hemorrhage, hypertensive disorders, infection, and complications from childbirth [5]. Cultural beliefs and the lack of awareness inhibit preparation in advance for delivery and expected babies in many developing countries. Since no action is taken before delivery, the family tries to act only when labor begins. In Ethiopia, the majority of pregnant women and families do not know how to recognize the dangerous signs of complications. This was exposed families to waste a great deal of time in recognizing the problem, getting organized, getting money, finding transport, and reaching to an appropriate referral facility after a complication occurs [6].

Good plans and preparations will increase the utilization of skilled care and reduce delays in accessing care in case of pregnancy and delivery complications [7]. However, inadequate preparation for rapid action in the event of obstetric complications, are well-documented factors contributing to delay in receiving skilled obstetric care in Ethiopia [8]. Even though BPCR is considered as the key strategy to enhance the utilization of skilled maternal care, no study was done to assess the practice of birth preparedness and complication readiness in the current study area. Therefore, this study is designed to measure the magnitude of birth preparedness and complication readiness practices and associated factors among pregnant mothers in Sibu Sire District, East Wollega Zone, Ethiopia.

\section{Methods and Materials}

\subsection{Study Design and Study Area}

A facility-based cross-sectional study design was conducted in Sibu Sire District, East Wollega Zone. Sibu Sire District is the western part of Ethiopia and is found at a distance of 50 kilometers from Nekemte town and 281 kilometers from the city town of Addis Ababa. According to the 2007 census of the Central Statistics Agency of Ethiopia, there were 138,869 populations in the district and 4,819 of them were pregnant women. The district contains 27 public health facilities (one district hospital, four health centers, and 22 health posts); from that one district hospital and four health centers were selected for the study.

\subsection{Study Population}

All pregnant mothers attending antenatal care at public health facilities were the source population, whereas 398 pregnant women attending ANC in selected public health facilities were included in our study population. Pregnant women who were seriously sick were excluded from the study.

\subsection{Sample Size and Sampling Procedure}

The sample size was determined by using a formula for the estimation of a single population proportion. The magnitude of BPCR practice was $41.1 \%(\mathrm{p}=0.411)$ [6]. Sample size computed using a 95\% confidence interval, 5\% margin of error (d), and $15 \%$ of the sample size was added for nonresponse. Using the correction formula the final sample size becomes 398 .

A systematic random sampling technique was applied to select the study subject. One district hospital and four health centers that provide basic packages of antenatal services were randomly selected by a lottery method. Out of 862 total monthly client flows, 398 samples were proportionally allocated to the five health facilities. Then, the studied pregnant women were interviewed every $\mathrm{k}^{\text {th }}$ of total clients.

\subsection{Operational Definition}

Birth preparedness (BP) is a preparation that is planned well before delivery. A woman is considered well prepared for birth if she has identified a place of delivery, skilled birth attendant, saved money, and identified the mode of transport ahead of delivery. A participant who fulfilled at least three of the above criteria was considered wellprepared. Complication readiness (CR) was defined as an awareness of pregnancy and delivery-related health problems and was considered present when a woman made all necessary plans for emergencies. For example, the arrangement of (funds, transport, blood donor, and designated decision maker) in case of hemorrhage and loss of consciousness [1, 17].

A skilled care provider/attendant is a professional caregiver who has the knowledge and skills to manage labor, childbirth, and the postpartum period. She must be able to recognize complications and refer the woman or the newborn to a higher level of care if the complications require intervention beyond skilled attendants' competence $[1,17]$.

The postpartum period was defined as a time starting from the delivery of the placenta up to 6 weeks. A newborn/neonatal period refers to the first 7 days of the newborn's life [1, 17].

Knowledge of BPCR:- A woman has knowledge if she 
knows five and above of the eleven selected components (identify place of delivery, save money, prepare essential items, identify skilled providers, awareness of the sign of an emergency, designating decision-makers on her, arranging emergency funds, identify the mode of transportation, arranging blood donors, identifying the institution for EmOC services) $[1,17]$.

Practice of BPCR:- A participant considered practiced if at least five of the selected components were done (identified place of delivery, saved money, prepared essential items, identified skilled providers, designated decision-makers on her, arranged emergency funds, identified mode of transportation, arranged bold donor, identified institution for EmOC service) [1, 17].

Attitude towards BPCR: Eight questions were asked on the attitude in a Likert-Scale. Pregnant mothers who scored whether above or equal to the mean score were labelled as favorable attitude and otherwise unfavorable attitude $[1,17]$.

\subsection{Data Collection Procedure}

Data was collected through a face-to-face interview with a structured questionnaire. The two-day training was given to two health officer supervisors and five diploma nurses to collect data For this study, a structured questionnaire was adopted from JHPIEGO, Monitoring BPCR and based on the conceptual framework after a thorough review of different kinds of literature [1]. The questionnaire was pre-tested for suitability before actual data collection in other facilities in $5 \%$ of the sample that had no chance of being included in this study.

\subsection{Data Quality Management}

The questionnaire was prepared in English, translated to Afan Oromo (local language) and back to English to keep the consistency of the question. Training of data collectors and supervisors and pretesting of the question was made to ensure the quality of data. The principal investigator and supervisors were present on the spot to check and review all completed questionnaires, and to ensure the completeness and consistency of the information collected.

\subsection{Data Processing and Analysis}

Data were coded and entered into EPI -INFO version7 and exported to SPSS version 21 for further analysis. Descriptive statistics using frequencies, proportions were presented in the table to characterize the participants. Bivariate regression was done and variables with $\mathrm{p} \leq 0.2$ were selected as a candidate for multivariable analyses. Multiple regression analysis using the odds ratio with its $95 \%$ confidence interval was employed to determine independent predictors. All statically tests were considered significant at alpha less than 0.05 .

\subsection{Ethical Consideration}

Ethical and study protocol approval was obtained from the Arsi University Institutional Research Review Committee. The purpose of the study, their right to refuse before and during the interview, and assurance of confidentiality were informed to the study participants. Privacy and anonymity of the individuals were ensured. Written informed consent was obtained before interviewing each participant.

\section{Results}

\subsection{Socio-demographic Characteristics}

A total of 398 pregnant women have participated in the study. Among the participants, 226 (56.8\%) had belonged to the age group of 25-34. About $246(61.8 \%)$ participants were from rural residents and $152(38.2 \%)$ were from urban residents. Most of the respondents were married (99.5\%), and more than half (56\%) were engaged in the farming occupation. Pregnant women who attended secondary and above school were $22.1 \%$; while $32.9 \%$ of the mothers had unable to read and write (Table 1).

Table 1. Socio-demographic characteristics of pregnant women attending antenatal care in health facilities in Sibu Sire District, Oromia Region, Ethiopia, 2020 (n=398).

\begin{tabular}{lll}
\hline Variable & Frequency & Percent \\
\hline Respondent age & & \\
$15-24$ & 113 & 28.4 \\
$25-34$ & 226 & 56.8 \\
35 and above & 59 & 14.8 \\
Place of Residence & & \\
Rural & 246 & 61.8 \\
Urban & 152 & 38.2 \\
Marital status & & \\
Married & 396 & 99.5 \\
Single & 1 & 0.25 \\
Divorce & 1 & 0.25 \\
Educational status of mothers & & \\
Unable to read and write & 131 & 32.9 \\
Read and write & 31 & 7.8 \\
Primary education & 148 & 37.2 \\
Secondary education and above & 88 & 22.1 \\
Occupation of mothers & & 28.5 \\
Housewife & 102 & 25.6 \\
Farmer & 223 & 52.5 \\
Merchant & 42 & 29.1 \\
Government employee & 30 & \\
Others & 1 & \\
Income & 116 & \\
$<1000$ Birr & & \\
1001-3000Birr & & \\
$\geq 3001 B i r r$ and above & & \\
\hline & & \\
\hline
\end{tabular}

\subsection{Obstetric Characteristics of the Respondent}

The study showed that $302(75.9 \%)$ of the respondents had more than two pregnancies. Participants who had up to $3^{\text {rd }}$ ANC visits were $52.3 \%$, whereas $47.7 \%$ of participants had four or more ANC visits. About 67 (16.8\%) respondents had a history of stillbirth (Table 2). 
Table 2. Obstetric characteristics of pregnant women attending antenatal care in health facilities in Sibu Sire District, Oromia Region, Ethiopia, 2020 ( $n=398$ ).

\begin{tabular}{|c|c|c|c|}
\hline Variable & Level & Frequency & Percent (\%) \\
\hline \multirow[t]{2}{*}{ Number of pregnancy } & 1 & 96 & 24.1 \\
\hline & 2 and above & 302 & 75.9 \\
\hline \multirow[t]{2}{*}{ Number of delivery } & 0 & 175 & 44,0 \\
\hline & 1 and above & 223 & 56.0 \\
\hline \multirow[t]{3}{*}{ Gestational age } & First trimester & 12 & 3.0 \\
\hline & Second trimester & 193 & 48.5 \\
\hline & Third trimester & 193 & 48.5 \\
\hline \multirow[t]{2}{*}{ Born alive } & 0 & 102 & 25.6 \\
\hline & 1 and above & 296 & 74.4 \\
\hline \multirow[t]{2}{*}{ Born dead } & 0 & 331 & 83.2 \\
\hline & 1 and above & 67 & 16.8 \\
\hline \multirow{2}{*}{ Number of ANC visit } & ANC 1 st visit to $3^{\text {rd }}$ visit & 208 & 52.3 \\
\hline & ANC $4^{\text {th }}$ and above visit & 190 & 47.7 \\
\hline
\end{tabular}

\subsection{Knowledge and Attitude Towards Birth Preparedness and Complication Readiness}

The proportion of pregnant women who know at least three danger signs during pregnancy, delivery, and postnatal period were $15.8 \%, 26.6 \%$, and $9.5 \%$, respectively. Severe vaginal bleeding has the most frequently mentioned complication by women during pregnancy (49.5\%), labor and delivery $(50 \%)$, and the postpartum period $(24.1 \%)$. The sources of information to hear about BPCR were health professionals $206(51.8 \%)$, friends/relatives $38(9.5 \%)$, and medias 19 (4.8\%). Of the total sampled respondents, $45.7 \%$ had more knowledge, and $42.5 \%$ of the respondents had a favorable attitude for BPCR (Table 3).

Table 3. Knowledge and Attitude of pregnant women attending antenatal care in health facilities in Sibu Sire District, Oromia Region, Ethiopia, 2020 (n=398).

\begin{tabular}{|c|c|c|c|}
\hline Variable & Yes/No & Frequency & Percent (\%) \\
\hline \multicolumn{4}{|l|}{ Knowledge } \\
\hline \multirow[t]{2}{*}{ Identify a place of delivery } & Yes & 284 & 71.4 \\
\hline & No & 114 & 28.6 \\
\hline \multirow[t]{2}{*}{ Save money } & Yes & 276 & 69.3 \\
\hline & No & 122 & 30.7 \\
\hline \multirow[t]{2}{*}{ Prepare essential items } & Yes & 269 & 67.3 \\
\hline & No & 129 & 32.4 \\
\hline \multirow[t]{2}{*}{ Identify skilled provider } & Yes & 116 & 29.1 \\
\hline & No & 282 & 70.9 \\
\hline \multirow[t]{2}{*}{ Awareness of the sign of an emergency } & Yes & 59 & 14.8 \\
\hline & No & 339 & 85.2 \\
\hline \multirow[t]{2}{*}{ Designating decision-maker on her } & Yes & 103 & 25.9 \\
\hline & No & 295 & 74.1 \\
\hline \multirow[t]{2}{*}{ Arranging emergency fund } & Yes & 138 & 34.7 \\
\hline & No & 260 & 65.3 \\
\hline \multirow[t]{2}{*}{ Identify the mode of transportation } & Yes & 62 & 15.6 \\
\hline & No & 336 & 84.4 \\
\hline \multirow[t]{2}{*}{ Arranging blood donors } & Yes & 17 & 4.3 \\
\hline & No & 381 & 95.7 \\
\hline \multirow[t]{2}{*}{ Identifying the institution for EmOC services } & Yes & 63 & 15.8 \\
\hline & No & 335 & 84.2 \\
\hline \multicolumn{4}{|l|}{ Summary of knowledge mentioned at least five variables from above } \\
\hline 5 and above (knowledgeable) & & 182 & 45.7 \\
\hline Less than 5 (not knowledgeable) & & 216 & 54.3 \\
\hline \multirow[t]{2}{*}{ Knowing at least three danger sign of pregnancy } & Yes & 63 & 15.8 \\
\hline & No & 335 & 84.2 \\
\hline \multirow[t]{2}{*}{ Knowing at least three danger sign of labor and delivery } & Yes & 106 & 26.6 \\
\hline & No & 292 & 73.4 \\
\hline \multirow[t]{2}{*}{ Knowing at least three danger sign of the postnatal period } & Yes & 38 & 9.5 \\
\hline & No & 360 & 90.5 \\
\hline \multirow[t]{2}{*}{ Having heard about birth preparedness and complication readiness } & Yes & 267 & 67.1 \\
\hline & No & 131 & 32.9 \\
\hline \multirow{2}{*}{ Attitude (above or equal to mean score) } & Yes & 169 & 42.5 \\
\hline & No & 229 & 57.5 \\
\hline
\end{tabular}




\subsection{The Magnitude of BPCR in the Study Setting}

The magnitude of BPCR practice in the study participants was $30.2 \%$ by preparation of five and/or more among eleven components (table 4).

Table 4. Magnitude of BPCR practice among pregnant women attending antenatal care in health facilities in Sibu Sire District, Oromia Region, Ethiopia, $2020(n=398)$.

\begin{tabular}{|c|c|c|c|}
\hline Variable & Level & Frequency & Percent (\%) \\
\hline \multicolumn{4}{|l|}{ Components of BPCR practice } \\
\hline Identified a place of delivery & Yes & 298 & 74.9 \\
\hline Saved money & Yes & 214 & 53.8 \\
\hline Prepared essential items & Yes & 244 & 61.3 \\
\hline Identified skilled provider & Yes & 111 & 27.9 \\
\hline Detected early sign of the emergency & Yes & 108 & 27.1 \\
\hline Designated decision-maker & Yes & 157 & 39.4 \\
\hline Identified the mode of transportation & Yes & 244 & 61.3 \\
\hline Arranged blood donor & Yes & 60 & 15.1 \\
\hline Identified an institution providing $24 \mathrm{hrs}$ EmOC service & Yes & 89 & 22.4 \\
\hline 5 and above (Good preparation) & & 120 & 30.2 \\
\hline Less than 5 (Poor preparation) & & 278 & 69.8 \\
\hline
\end{tabular}

\subsection{Factors Associated with Birth Preparedness and Complication Readiness Practice}

From mothers' educational status, pregnant women who attended primary school, secondary, and above school, respectively, were 2.5 times $[\mathrm{AOR}=2.5(95 \% \mathrm{CI} ; 1.30,5.18)]$ and 5.8 times $[\mathrm{AOR}=5.8(95 \% \mathrm{CI} ; 2.57,13.46)]$ more likely to be prepared for birth and complication compared to women who had no any formal education. Women who had a history of stillbirth were statistically significant associations $[\mathrm{AOR}=1.97 \quad(95 \% \quad \mathrm{CI} ; 1.01,3.86)]$ with birth and complication readiness practice. ANC visits during the $4^{\text {th }}$ and above were 2.1 times [AOR=2.11 $(95 \% \mathrm{CI} ; 1.25,3.55)$ ] more to be prepared for birth and complications than those attended below the fourth visit (table 5).

Table 5. Multivariate logistic regression analysis of birth preparedness and complication readiness practice among pregnant women attending antenatal care in health facilities in Sibu Sire District, 2020 (398).

\begin{tabular}{|c|c|c|c|c|}
\hline \multicolumn{5}{|l|}{ BP/CR } \\
\hline Variables & Yes n $(\%)$ & No n (\%) & COR $(95 \% C I)$ & AOR (95\%CI) \\
\hline \multicolumn{5}{|l|}{ Residence } \\
\hline Rural & $65(54.2)$ & $181(65.1)$ & 1 & 1 \\
\hline Urban & $55(45.8)$ & $97(34.9)$ & $1.57(1.02-2.44)$ & $1.14(0.62-2.10)$ \\
\hline \multicolumn{5}{|c|}{ Educational status of mothers } \\
\hline Not educated & $24(20)$ & $107(38.5)$ & 1 & 1 \\
\hline Read and write & $8(6.7)$ & $23(8.3)$ & $1.55(0.61-3.88)$ & $2.02(0.72-5.68)$ \\
\hline Primary education & $42(35)$ & $106(38.1)$ & $1.76(1.00-3.12)$ & $2.59(1.30-5.18)$ \\
\hline Secondary and above & $46(38.3)$ & $42(15.1)$ & $4.88(2.65-8.97)$ & $5.88(2.57-13.46)$ \\
\hline \multicolumn{5}{|l|}{ Family income } \\
\hline$<1000$ ETB & $25(20.8)$ & $88(31.7)$ & 1 & 1 \\
\hline 1000-3000ЕTB & $55(45.8)$ & $114(41)$ & $1.69(0.98-2.93)$ & $1.17(0.61-2.24)$ \\
\hline 3001ETB and above & $40(33.3)$ & $76(27.3)$ & $1.85(1.03-3.33)$ & $0.63(0.28-1.42)$ \\
\hline \multicolumn{5}{|l|}{ Total gave birth } \\
\hline 0 & $29(24.2)$ & $146(52.5)$ & 1 & 1 \\
\hline 1 and above & $91(75.8)$ & $132(47.5)$ & $3.47(2.14-5.60)$ & $4.31(2.45-7.60)$ \\
\hline \multicolumn{5}{|l|}{ History of still birth } \\
\hline 0 & $87(72.5)$ & $244(87.8)$ & 1 & 1 \\
\hline 1 and above & $33(27.5)$ & $34(12.2)$ & $2.72(1.59-4.66)$ & $1.97(1.01-3.86)$ \\
\hline \multicolumn{5}{|l|}{ ANC visit } \\
\hline $1^{\text {st }}$ visit to $3^{\text {rd }}$ visit & $49(40.8)$ & $159(57.2)$ & 1 & 1 \\
\hline 4th and above visit & $71(59.2)$ & $119(42.8)$ & $1.93(1.25-2.99)$ & $2.11(1.25-3.55)$ \\
\hline \multicolumn{5}{|c|}{ Knowledge of at least three danger sign of pregnancy } \\
\hline Yes & $30(25)$ & $41(14.7)$ & $1.92(1.13-3.27)$ & $2.21(1.17-4.18)$ \\
\hline No & $90(75)$ & $237(85.3)$ & 1 & 1 \\
\hline \multicolumn{5}{|c|}{ Knowledge of at least three danger sign of the postnatal period } \\
\hline Yes & $17(14.2)$ & $21(7.6)$ & $2.02(1.02-3.98)$ & $2.00(0.84-4.72)$ \\
\hline No & $103(85.8)$ & $257(92.4)$ & 1 & 1 \\
\hline \multicolumn{5}{|l|}{ Heard BPCR } \\
\hline Yes & $69(57.5)$ & $198(71.2)$ & 1 & 1 \\
\hline No & $51(42.5)$ & $80(28.8)$ & $1.82(1.17-2.85)$ & $2.55(1.48-4.40)$ \\
\hline Knowledge of BPCR & & & & \\
\hline
\end{tabular}




\begin{tabular}{lllll}
\hline BP/CR & & & & \\
\hline Variables & Yes n (\%) & No n (\%) & COR (95\%CI) & AOR (95\%CI) \\
\hline Yes & $66(36.5)$ & $116(63.3)$ & $1.70(1.10-2.62)$ & $2.1(1.24-3.53)$ \\
No & $54(25)$ & $162(75)$ & 1 & 1 \\
Attitude of BPCR & & & \\
Favorable & $60(50)$ & $109(39.2)$ & $1.55(1.00-2.38)$ & $1.41(0.84-2.37)$ \\
Unfavorable & $60(50)$ & $169(60.8)$ & 1 & 1 \\
\hline
\end{tabular}

\section{Discussion}

The magnitude of BPCR practice among pregnant women in Sibu Sire district was $30.2 \%$. This magnitude was low when compared to the study done in India (41\%), Central Tanzania (58.2\%), and Nigeria (40.3\%) [9-11]. Reasons for the difference may be due to the countries are better wealth status and availability and accessibility of health services for intervention than in Ethiopia. However, it was consistent with studies conducted in Arbaminch Zura Woreda of Sidama Zone (30\%) [12]. On the other hand, this finding was higher compared to the study done in Basoliben district $(26.9 \%)$ and Duguna Fango district $(18.3 \%)[13,14]$. The possible reason might be the difference in the study setting.

Determinants of birth preparedness and complication readiness practice

Using multivariable regression analysis, mothers' educational status was statistically significantly associated with BPCR. Pregnant women who attended primary school, secondary, and above were more likely to have BPCR with $[\mathrm{AOR}=2.5(95 \% \mathrm{CI} ; 1.30,5.18)],[\mathrm{AOR}=5.8(95 \% \mathrm{CI} ; 2.57$, 13.46)] respectively when compared to counterparts. This finding was consistent with the results from India and Arsi Robe of Ethiopia, where the magnitude of BPCR was significantly higher among educated mothers $[15,16]$.

History of stillbirth was another independent predictor that was statistically associated with BPCR practice. Women who had a history of stillbirth were had more odds for BPCR than without a history of stillbirth [AOR=1.97 (95\% C/I; 1.01, 3.86)]. This result is comparable to the findings of studies conducted in Adegrat and Adama town $[17,18]$. The possible reason for this result might be due to those pregnant women could anticipate serious complications from their previous experiences. There was also an association between the number of ANC visits and BPCR practice. Pregnant women who attended ANC visits 4 times and above were more to be planned for BPCR than those who attended below fourth visit $[\mathrm{AOR}=2.11(95 \% \mathrm{CI} ; 1.25$, 3.55)]. A similar report from Adegrat showed that ANC visit at $4^{\text {th }}$ and above was significantly associated with BPCR [17]. The evidence is suggested by WHO that ANC visit is more effective if pregnant woman visit a minimum of four during her total pregnancy time.

Knowing to mention three and above of at least three danger signs during pregnancy was another determinant that statistically significantly associated with BPCR. Mothers who knew mentioning three and above for at least three danger signs during pregnancy were more to be prepared for BPCR than those who had mentioned below three
$[\mathrm{AOR}=2.21(95 \% \mathrm{C} / \mathrm{I} ; 1.17,4.18)]$. This finding was in line with studies in Arsi Robe woreda and South wollo [16, 19]. Besides, Pregnant women that had adequate knowledge were two times more likely to have BPCR than those who lacked knowledge $[\mathrm{AOR}=2.1(95 \% \mathrm{CI} ; 1.24,3.53)]$. The result was consistent with a report from South Wollo and Goba woreda $[19,20]$. This implies that mothers with knowledge of obstetric complications may predict something that happens during pregnancy and seek advice and support from health personnel.

The hundred percent response rate and the use of primary data were the strength of the study. However, the readers of this article should bear in mind that the study limitation is inherent to the design and the finding implies the single time fact. The smallness of samples and place also can limit the representativeness of the finding.

\section{Conclusions and Recommendations}

The findings of the study indicated that a low magnitude of BPCR practice was observed among pregnant women of Sibu Sire district when we compared to the study findings in different parts of the world. The analysis of the data shows that from multiple evaluated variables some of them attending primary school, secondary, and above, having ANC visits of 4 times and above, and having a history of stillbirth were had a statistically significant association. Having adequate knowledge and hearing about BPCR were also important positive predictors in reducing the magnitude of obstetric complications in pregnant women in the study setting.

From the findings of this study, authors recommend that increasing awareness of BPCR for pregnant women should also be considered as an important intervention program. Furthermore, there is a need to conduct similar researches in private health facilities at a large scale and using both qualitative and quantitative methods.

\section{Abbreviations}

ANC, ante-natal care; AOR, adjusted odds ratio; BPCR, birth preparedness and complication readiness; $\mathrm{CI}$, confidence interval; COR, crude odds ratio; EDHS, Ethiopian demographic and health survey; EmOC, emergency obstetric care; ETB, Ethiopian birr; SPSS, statistical package for social science; WHO, a world health organization.

\section{Availability of Data}

Data is available and kept in the investigators' database. Can only be shared at reasonable request. 


\section{Ethical Issues}

Ethical and study protocol approval was obtained from the Arsi University College of Health Sciences Research Review Committee. Written informed consent was obtained from each participant with the agreement to participate just before starting interviewing.

\section{Author Contributions}

All authors made considerable contributions to the conception, design, and execution, acquisition of data, or analysis and interpretation of data; had a part in drafting the article, or revised it critically; agreed to submit to the journal; gave final approval for the version to be published; and agreed to be responsible and accountable for all regards of the articles.

\section{Disclosure}

All the authors do not have any possible conflicts of interest.

\section{Acknowledgements}

The authors would like to thank data collectors, supervisors, and respondents for their willingness and participation in the study. Our appreciation also goes to Arsi University and Oromia Health Bureau for their facilitation.

\section{References}

[1] JHPIEGO (2004). Monitoring Tools Birth Preparedness \& Complication Readiness (BPCR). p. 338.

[2] WHO (2015). WHO recommendations on health promotion interventions for maternal and new born health; 1-41.

[3] Mendez A, \& Sawan M. (2011). Chronic monitoring of bladder volume: A critical review and assessment of measurement methods. Can J Urol; 18 (1): 5504-16.

[4] Centeral Statistical Agency (CSA) Ethiopia and ICF (2016). Ethiopian Demographic and Health Survey 206. Addis Ababa, Ethiopia.

[5] Partnership for Maternal N \& CH, Member. (2017). Progress on the Every Woman Every Child Global Strategy; 1-79. Available from: http://www.who.int/pmnch/activities/advocacy/globalstrategy/ 2016_2030/gspr/en/.

[6] Begashaw B, Tesfaye Y, Zelalem E, Ubong U, Kumalo A. (2017). Assessment of Birth Preparedness and Complication Readiness among Pregnant Mothers Attending Ante Natal Care Service in Mizan-Tepi University Teaching Hospital, South West Ethiopia. Clin Mother Child Heal; 14 (1): 1-7.

[7] Debelew GT, Afework F, Yalew AW. (2014). Factors affecting birth preparedness and complication readiness in Jimma Zone, Southwest Ethiopia: A multilevel analysis. Pan Afr Med J; 19: 1-14.
[8] UNFPA (2017). The maternal health themetic fund: Towards Equality in Access, Quality of Care and Accountability. Available from: https:/www.unfpa.org/sites/default/files/pubpdf/51375_MHTF_AnnualReport_web.pdf.

[9] Acharya AS, Kaur R, Prasuna JG, Rasheed N. (2015). Making Pregnancy Safer - Birth Preparedness and Complication Readiness Study among Antenatal Women Attendees of A Primary Health Center, Delhi. Indian J Community Med; 40 (2): $127-34$.

[10] Bintabara D, Mohamed MA, Mghamba J, Wasswa P, Mpembeni RNM. (2015). Birth preparedness and complication readiness among recently delivered women in chamwino district, central Tanzania: A cross sectional study "Obstetrics." Reprod Health; 12 (1): 1-8.

[11] Idowu A (2015). Birth Preparedness and Complication Readiness among Women Attending Antenatal Clinics in Ogbomoso, South West, Nigeria. Int J MCH AIDS; 4 (1): 47 56 .

[12] Andarge E, Nigussie A, Wondafrash M. (2017). Factors associated with birth preparedness and complication readiness in Southern Ethiopia: a community based cross-sectional study. BMC Pregnancy Childbirth; 17 (1): 412.

[13] Worku WB (2014). Birth Preparedness and Complication Readiness and Associated Factors among Pregnant Women in Basoliben District, Amhara Regional State, Northwest Ethiopia. Prim Heal Care Open Access; 04 (04): 2-7.

[14] Gebre M, Gebremariam A, Abebe TA. (2015). Birth preparedness and complication readiness among pregnant women in Duguna Fango District, Wolayta Zone, Ethiopia. PLoS One; 10 (9): 1-12.

[15] Akshaya KM, \& Shivalli S. (2017). Birth preparedness and complication readiness among the women beneficiaries of selected rural primary health centers of Dakshina Kannada district, Karnataka, India. PLoS One; 12 (8): 1-15.

[16] Kaso M, \& Addisse M. (2014). Birth preparedness and complication readiness in Robe Woreda, Arsi Zone, Oromia Region, Central Ethiopia: A cross-sectional study. Reprod Health; 11 (1): 1-12.

[17] Mihret H, \& Mesganaw F. (2008). Birth preparedness and complication readiness among women in Adigrat town, northern Ethiopia. Ethiopian journal of health Development; 22: 14-20.

[18] Girmay A (2016). Assessment of Magnitude and Factors Associated with Birth Preparedness and Complication Readiness Among Pregnant Women Attending Antenatal Clinic of Adama Town Health Facilities, Central Ethiopia. Eur J Prev Med; 4 (2): 32.

[19] Bitew Y, Awoke W, Chekol S. (2016). Birth Preparedness and Complication Readiness Practice and Associated Factors among Pregnant Women, Northwest Ethiopia. Int Sch Res Not; 2016: $1-8$.

[20] Markos D, \& Bogale D. (2014). Birth preparedness and complication readiness among women of child bearing age group in Goba woreda, Oromia region, Ethiopia. BMC Pregnancy Childbirth; 14 (1): 1-9. 